\title{
Steiner Degree Distance of Two Graph Products
}

\author{
Yaping Mao, Zhao Wang, Kinkar Ch. Das
}

\begin{abstract}
The degree distance $D D(G)$ of a connected graph $G$ was invented by Dobrynin and Kochetova in 1994. Recently, one of the present authors introduced the concept of $k$-center Steiner degree distance defined as

$$
S D D_{k}(G)=\sum_{\substack{S \subseteq V(G) \\|S|=k}}\left[\sum_{v \in S} \operatorname{deg}_{G}(v)\right] d_{G}(S),
$$

where $d_{G}(S)$ is the Steiner $k$-distance of $S$ and $\operatorname{deg}_{G}(v)$ is the degree of the vertex $v$ in $G$. In this paper, we investigate the Steiner degree distance of complete and Cartesian product graphs.
\end{abstract}

\section{Introduction}

All graphs in this paper are assumed to be undirected, finite, and simple. Let $G$ be such a graph with vertex set $V(G)$ and edge set $E(G)$. Then the order and size of $G$ are $n=n(G)=|V(G)|$ and $m=m(G)=|E(G)|$. In

Key Words: Steiner degree distance, Degree distance, Complete product, Cartesian product.

2010 Mathematics Subject Classification: Primary 05C07; Secondary 05C90.

Corresponding author (Kinkar Ch. Das)

Received: 10.04 .2018 .

Accepted: 30.07. 2018 
other words, $G$ has $n$ vertices and $m$ edges. The degree $\operatorname{deg}_{G}(u)$ of the vertex $u \in V(G)$ is number of first neighbors of this vertex.

Distance is one of the basic concepts of graph theory [7]. If $G$ is connected and $u, v \in V(G)$, then the distance $d(u, v)=d_{G}(u, v)$ between $u$ and $v$ is the length of a shortest path connecting $u$ and $v$. If $v$ is a vertex of a connected graph $G$, then the eccentricity $\varepsilon(v)$ of $v$ is defined as $\varepsilon(v)=\max \{d(u, v) \mid u \in$ $V(G)\}$. Furthermore, the radius $\operatorname{rad}(G)$ and $\operatorname{diameter} \operatorname{diam}(G)$ of $G$ are $\operatorname{rad}(G)=\min \{\varepsilon(v) \mid v \in V(G)\}$ and $\operatorname{diam}(G)=\max \{\varepsilon(v) \mid v \in V(G)\}$. These latter two quantities are related by the inequalities $\operatorname{rad}(G) \leq \operatorname{diam}(G) \leq$ $2 \operatorname{rad}(G)$. More details on this subject can be found in [14]. We refer to [5] for graph theoretical notation and terminology not specified here.

For a graph $G$ with vertex set $V(G)$, the degree distance is defined as [13]

$$
D D=D D(G)=\sum_{\{u, v\} \in V(G)}\left[\operatorname{deg}_{G}(u)+\operatorname{deg}_{G}(v)\right] d_{G}(u, v)
$$

For more details on degree-and-distance-based graph invariant, we refer to $[2,3,4,6,17,18,30,32,35]$.

The Wiener index $W(G)$ of the graph $G$ is defined as

$$
W(G)=\sum_{\{u, v\} \subseteq V(G)} d_{G}(u, v) .
$$

Details on this oldest distance-based topological index can be found in numerous surveys, e.g., in $[11,12,19,20,33,34,36]$.

The Steiner distance of a graph, introduced by Chartrand et al. [9] in 1989, is a natural and nice generalization of the concept of classical graph distance. For a subset $S$ of the vertex set $V(G)$, consisting of at least two vertices, the Steiner distance $d(S)$ (or simply the distance of $S$ ) is the minimum size (number of edges) of a connected subgraphs whose vertex set contains $S$. This connected subgraph is necessarily a tree and is referred to as a Steiner tree. 
Note that if $S=\{u, v\}$, then $d(S)=d(u, v)$ is nothing new, but the classical distance between $u$ and $v$. Clearly, if $|S|=k$, then $d(S) \geq k-1$.

Let $n$ and $k$ be integers such that $2 \leq k \leq n$. The Steiner $k$-eccentricity $\varepsilon_{k}(v)$ of a vertex $v$ of $G$ is $\varepsilon_{k}(v)=\max \{d(S)|S \subseteq V(G)| S \mid,=k$, and $v \in S\}$. The Steiner k-radius of $G$ is $\operatorname{srad}_{k}(G)=\min \left\{\varepsilon_{k}(v) \mid v \in V(G)\right\}$, whereas the Steiner $k$-diameter of $G$ is $\operatorname{sdiam}_{k}(G)=\max \left\{\varepsilon_{k}(v) \mid v \in V(G)\right\}$. Note that for every connected graph $G, \varepsilon_{2}(v)=\varepsilon(v)$ for all vertices $v$ of $G, \operatorname{srad}_{2}(G)=$ $\operatorname{rad}(G)$ and $\operatorname{sdiam}_{2}(G)=\operatorname{diam}(G)$. For more details on Steiner distance, we refer to $[1,8,9,10,14,31]$.

The following result is immediate.

Observation 1.1. If $H$ is a connected spanning subgraph of $G$, then

$$
\operatorname{siam}_{k}(G) \leq \operatorname{siam}_{k}(H)
$$

holds for all $k, 2 \leq k \leq n$.

Li et al. [21] put forward a Steiner-distance-based generalization of the Wiener index concept. According to [21], the k-center Steiner Wiener index $S W_{k}(G)$ of the graph $G$ is defined by

$$
S W_{k}(G)=\sum_{\substack{S \subseteq V(G) \\|S|=k}} d(S) .
$$

For $k=2$, the above defined Steiner Wiener index coincides with the ordinary Wiener index, Eq. (2). It is usual to consider $S W_{k}$ for $2 \leq k \leq n-1$, but the above definition would be applicable also in the cases $k=1$ and $k=n$, implying $S W_{1}(G)=0$ and $S W_{n}(G)=n-1$. For more details on Steiner Wiener index, we refer to $[21,22,26,27,28,29]$.

Recently, Gutman [16] offered an analogous generalization of the concept of degree distance, Eq. (1). Thus, the $k$-center Steiner degree distance $S D D_{k}(G)$ 
of $G$ is defined as

$$
S D D_{k}(G)=\sum_{\substack{S \subseteq V(G) \\|S|=k}}\left[\sum_{v \in S} \operatorname{deg}_{G}(v)\right] d_{G}(S) .
$$

Mao et al. [28] reported expressions for the $k$-center Steiner degree distance of the star, path, as well as the complete and complete bipartite graphs, and got general expressions for $S D D_{k}(G)$ for $k=n, n-1$. They also obtained sharp lower and upper bounds for $S D D_{k}$. Very recently, Mao and Das [25] generalize the concept of Gutman index by Steiner distance. The Steiner Gutman $k$-index $S G u t_{k}(G)$ of graph $G$ is defined by

$$
S G u t_{k}(G)=\sum_{\substack{S \subseteq V(G) \\|S|=k}}\left[\prod_{v \in S} \operatorname{deg}_{G}(v)\right] d_{G}(S)
$$

The join and Cartesian products are defined as follows:

The join or complete product $G \vee H$ of two disjoint graphs $G$ and $H$, is the graph with vertex set $V(G) \cup V(H)$ and edge set $E(G) \cup E(H) \cup\{u v \mid u \in$ $V(G), v \in V(H)\}$.

The Cartesian product $G \square H$ of two graphs $G$ and $H$, is the graph with vertex set $V(G) \times V(H)$, in which two vertices $(u, v)$ and $\left(u^{\prime}, v^{\prime}\right)$ are adjacent if and only if $u=u^{\prime}$ and $v v^{\prime} \in E(H)$, or $v=v^{\prime}$ and $u u^{\prime} \in E(G)$.

\section{Complete product}

In this section, we give the exact expression of $S D D_{k}(G \vee H)$.

Theorem 2.1. Let $G, H$ be two connected graphs of order $a, b(a \leq b)$, respectively. Let $k$ be an integer with $3 \leq k \leq a+b$. 
(1) If $1 \leq k \leq a$, then

$$
\begin{aligned}
S D D_{k}(G \vee H)= & k\left(\begin{array}{l}
a \\
k
\end{array}\right)-x-2 e(G)\left(\begin{array}{l}
a-1 \\
k-1
\end{array}\right)-\sum_{i=1}^{x}\left(\sum_{v \in S_{i}} \operatorname{deg}_{G}(v)\right) \\
& +k\left(\begin{array}{l}
b \\
k
\end{array}\right)-y-2 e(H)\left(\begin{array}{c}
b-1 \\
k-1
\end{array}\right)-\sum_{i=1}^{y}\left(\sum_{v \in S_{i}^{\prime}} \operatorname{deg}_{H}(v)\right) \\
& +2(k-1)[e(G)+e(H)+a b]\left(\begin{array}{c}
a+b-1 \\
k-1
\end{array}\right) \\
& -(k-1) a b\left(\begin{array}{c}
a-1 \\
k-1
\end{array}\right)-(k-1) a b\left(\begin{array}{c}
b-1 \\
k-1
\end{array}\right),
\end{aligned}
$$

where $S_{1}, S_{2}, \ldots, S_{x}$ are all the $k$-subsets of $V(G)$ such that $G\left[S_{i}\right](1 \leq i \leq$ $x)$ is connected, and $S_{1}^{\prime}, S_{2}^{\prime}, \ldots, S_{y}^{\prime}$ are all the $k$-subsets of $V(H)$ such that $H\left[S_{i}^{\prime}\right](1 \leq i \leq y)$ is connected.

(2) If $a<k \leq b$, then

$$
\begin{aligned}
S D D_{k}(G \vee H) & =a k^{2}\left(\begin{array}{l}
b \\
k
\end{array}\right)-a k x+2 e(H)\left(\begin{array}{c}
b-1 \\
k-1
\end{array}\right)-\sum_{i=1}^{x}\left(\sum_{v \in S_{i}} \operatorname{deg}_{H}(v)\right) \\
& +2(k-1)[e(G)+e(H)+a b]\left(\begin{array}{c}
a+b-1 \\
k-1
\end{array}\right)-(k-1) a b\left(\begin{array}{l}
a-1 \\
k-1
\end{array}\right) \\
& -(k-1) a b\left(\begin{array}{l}
b-1 \\
k-1
\end{array}\right)-(k-1) \cdot 2 e(G)\left(\begin{array}{l}
a-1 \\
k-1
\end{array}\right),
\end{aligned}
$$

where $S_{1}, S_{2}, \ldots, S_{x}$ are all the $k$-subsets of $V(H)$ such that $H\left[S_{i}\right](1 \leq i \leq x)$ is connected.

(3) If $b<k \leq a+b$, then

$$
\begin{gathered}
S D D_{k}(G \vee H)=2(k-1) e(G)\left[\left(\begin{array}{c}
a+b-1 \\
k-1
\end{array}\right)-\left(\begin{array}{c}
a-1 \\
k-1
\end{array}\right)\right]-(k-1) a b\left[\left(\begin{array}{l}
a-1 \\
k-1
\end{array}\right)\right. \\
\left.+\left(\begin{array}{c}
b-1 \\
k-1
\end{array}\right)\right]+2(k-1) e(H)\left[\left(\begin{array}{c}
a+b-1 \\
k-1
\end{array}\right)-\left(\begin{array}{c}
b-1 \\
k-1
\end{array}\right)\right] .
\end{gathered}
$$


Proof. Let $V(G)=\left\{u_{1}, u_{2}, \ldots, u_{a}\right\}$ and $V(H)=\left\{v_{1}, v_{2}, \ldots, v_{b}\right\}$. Without loss of generality, we can assume that $a \leq b$. We distinguish the following three cases to show this theorem.

Case 1. $1 \leq k \leq a$. For any $S \subseteq V(G)$ and $|S|=k$, we have $S \cap V(G)=\varnothing$, or $S \cap V(H)=\varnothing$, or $S \cap V(G) \neq \varnothing$ and $S \cap V(H) \neq \varnothing$.

If $S \cap V(H)=\varnothing$, then $S \subseteq V(G)$. Recall that $S_{1}, S_{2}, \ldots, S_{\left(\begin{array}{c}a \\ k\end{array}\right)}$ be all the $k$-subsets of $V(G)$, and $S_{1}, S_{2}, \ldots, S_{x}$ are all the $k$-subsets of $V(G)$ such that $G\left[S_{i}\right](1 \leq i \leq x)$ is connected. Then $d_{G}\left(S_{i}\right)=d_{G \vee H}\left(S_{i}\right)=k-1$ for each $i(1 \leq i \leq x)$. For each $i\left(x+1 \leq i \leq\left(\begin{array}{l}a \\ k\end{array}\right)\right)$, we have $d_{G}\left(S_{i}\right) \geq k$ and $d_{G \vee H}\left(S_{i}\right)=k$. For $v \in V(G \vee H)$, if $v \in V(G)$, then $\operatorname{deg}_{G \vee H}(v)=\operatorname{deg}_{G}(v)+b$.

This case contributes to $S D D_{k}(G \vee H)$ by

$$
\begin{aligned}
& \sum_{\substack{S \subseteq \vee(G) \\
|S|=k}}\left(\sum_{v \in S} \operatorname{deg}_{G \vee H}(v)\right) d_{G \vee H}(S) \\
& =\sum_{\substack{S \subseteq V(G) \\
|S|=k}}\left(\sum_{v \in S}\left(\operatorname{deg}_{G}(v)+b\right)\right) d_{G \vee H}(S) \\
& =\sum_{\substack{S \subseteq V(G) \\
|S|=k}}\left(\sum_{v \in S} \operatorname{deg}_{G}(v)\right) d_{G \vee H}(S)+b k \sum_{\substack{S \subseteq Y(G) \\
|S|=k}} d_{G \vee H}(S) \\
& =(k-1) \sum_{\substack{S \subseteq V(G),|S|=k \\
d_{G}(S)=k-1}}\left(\sum_{v \in S} \operatorname{deg}_{G}(v)\right)+k \sum_{\substack{S \subseteq V(G),|S|=k \\
d_{G}(S) \geq k}}\left(\sum_{v \in S} \operatorname{deg}_{G}(v)\right)+b k \sum_{\substack{S \subseteq V(G) \\
|S|=k}} d_{G \vee H}(S) \\
& =k \sum_{\substack{S \subseteq V(G) \\
|S|=k}}\left(\sum_{v \in S} \operatorname{deg}_{G}(v)\right)-\sum_{\substack{S \subseteq V(G),|S|=k \\
d_{G}(S)=k-1}}\left(\sum_{v \in S} \operatorname{deg}_{G}(v)\right)+b k\left[k\left(\begin{array}{l}
a \\
k
\end{array}\right)-x\right] \\
& =2 k e(G)\left(\begin{array}{l}
a-1 \\
k-1
\end{array}\right)-\sum_{i=1}^{x}\left(\sum_{v \in S_{i}} \operatorname{deg}_{G}(v)\right)+b k^{2}\left(\begin{array}{l}
a \\
k
\end{array}\right)-b k x .
\end{aligned}
$$


If $S \cap V(G)=\varnothing$, then $S \subseteq V(H)$. Similarly, this case contributes to $S D D_{k}(G \vee$ $H)$ by

$$
2 k e(H)\left(\begin{array}{l}
b-1 \\
k-1
\end{array}\right)-\sum_{i=1}^{y}\left(\sum_{v \in S_{i}^{\prime}} \operatorname{deg}_{H}(v)\right)+a k^{2}\left(\begin{array}{l}
b \\
k
\end{array}\right)-a k y .
$$

Suppose $S \cap V(G) \neq \varnothing$ and $S \cap V(H) \neq \varnothing$. In this case, $d_{G \vee H}(S)=k-1$.

This case contributes to $S D D_{k}(G \vee H)$ by

$$
\begin{aligned}
& \sum_{\substack{S \subseteq V(G),|S|=k \\
S \cap V(G) \neq \varnothing, S \cap V(H) \neq \varnothing}}\left(\sum_{v \in S} \operatorname{deg}_{G \vee H}(v)\right) d_{G \vee H}(S) \\
= & (k-1) \sum_{\substack{S \subseteq V(G),|S|=k \\
S \cap V(G) \neq \varnothing, S \cap V(H) \neq \varnothing}}\left(\sum_{v \in S} \operatorname{deg}_{G \vee H}(v)\right) \\
= & (k-1) \cdot 2 e(G \vee H)\left(\begin{array}{c}
a+b-1 \\
k-1
\end{array}\right)-(k-1) \cdot 2 e(G)\left(\begin{array}{l}
a-1 \\
k-1
\end{array}\right) \\
& -(k-1) a b\left(\begin{array}{l}
a-1 \\
k-1
\end{array}\right)-(k-1) \cdot 2 e(H)\left(\begin{array}{l}
b-1 \\
k-1
\end{array}\right)-(k-1) a b\left(\begin{array}{l}
b-1 \\
k-1
\end{array}\right) \\
= & (k-1)(2 e(G)+2 e(H)+2 a b)\left(\begin{array}{c}
a+b-1 \\
k-1
\end{array}\right)-(k-1) \cdot 2 e(G)\left(\begin{array}{l}
a-1 \\
k-1
\end{array}\right) \\
& -(k-1) a b\left(\begin{array}{l}
a-1 \\
k-1
\end{array}\right)-(k-1) \cdot 2 e(H)\left(\begin{array}{l}
b-1 \\
k-1
\end{array}\right)-(k-1) a b\left(\begin{array}{l}
b-1 \\
k-1
\end{array}\right) .
\end{aligned}
$$

From the above arguments, we conclude that

$$
\begin{array}{r}
S D D_{k}(G \vee H)=\quad b k^{2}\left(\begin{array}{l}
a \\
k
\end{array}\right)-b k x+2 e(G)\left(\begin{array}{l}
a-1 \\
k-1
\end{array}\right)-\sum_{i=1}^{x}\left(\sum_{v \in S_{i}} \operatorname{deg}_{G}(v)\right) \\
+a k^{2}\left(\begin{array}{l}
b \\
k
\end{array}\right)-a k y+2 e(H)\left(\begin{array}{l}
b-1 \\
k-1
\end{array}\right)-\sum_{i=1}^{y}\left(\sum_{v \in S_{i}^{\prime}} \operatorname{deg}_{H}(v)\right) \\
+2(k-1)[e(G)+e(H)+a b]\left(\begin{array}{c}
a+b-1 \\
k-1
\end{array}\right)-(k-1) a b\left(\begin{array}{l}
a-1 \\
k-1
\end{array}\right)-(k-1) a b\left(\begin{array}{l}
b-1 \\
k-1
\end{array}\right),
\end{array}
$$


where $S_{1}, S_{2}, \ldots, S_{x}$ are all the $k$-subsets of $V(G)$ such that $G\left[S_{i}\right](1 \leq i \leq$ $x)$ is connected, and $S_{1}^{\prime}, S_{2}^{\prime}, \ldots, S_{y}^{\prime}$ are all the $k$-subsets of $V(H)$ such that $H\left[S_{i}^{\prime}\right](1 \leq i \leq y)$ is connected.

Case 2. $a<k \leq b$. For any $S \subseteq V(G)$ and $|S|=k$, we have $S \cap V(G)=\varnothing$, or $S \cap V(G) \neq \varnothing$ and $S \cap V(H) \neq \varnothing$.

If $S \cap V(G)=\varnothing$, then $S \subseteq V(H)$. Recall that $S_{1}, S_{2}, \ldots, S_{\left(\begin{array}{c}b \\ k\end{array}\right)}$ be all the $k$-subsets of $V(H)$, and $S_{1}, S_{2}, \ldots, S_{x}$ are all the $k$-subsets of $V(H)$ such that $G\left[S_{i}\right](1 \leq i \leq x)$ is connected. Then $d_{H}\left(S_{i}\right)=d_{G \vee H}\left(S_{i}\right)=k-1$ for each $i(1 \leq i \leq x)$. For each $i\left(x+1 \leq i \leq\left(\begin{array}{l}b \\ k\end{array}\right)\right)$, we have $d_{H}\left(S_{i}\right) \geq k$ and $d_{G \vee H}\left(S_{i}\right)=k$. For $v \in V(G \vee H)$, if $v \in V(H)$, then $\operatorname{deg}_{G \vee H}(v)=\operatorname{deg}_{H}(v)+a$.

This case contributes to $S D D_{k}(G \vee H)$ by

$$
\begin{aligned}
& \sum_{\substack{S \subseteq V(H) \\
|S|=k}}\left(\sum_{v \in S} \operatorname{deg}_{G \vee H}(v)\right) d_{G \vee H}(S) \\
= & \sum_{\substack{S \subseteq V(H) \\
|S|=k}}\left(\sum_{v \in S}\left(\operatorname{deg}_{H}(v)+a\right)\right) d_{G \vee H}(S) \\
= & \sum_{\substack{S \subseteq V(H) \\
|S|=k}}\left(\sum_{v \in S} \operatorname{deg}_{H}(v)\right) d_{G \vee H}(S)+a k \sum_{\substack{S \subseteq V(H) \\
|S|=k}} d_{G \vee H}(S) \\
= & (k-1) \sum_{\substack{S \subseteq V(H),|S|=k \\
\bar{d}_{H}(S)=k-1}}\left(\sum_{v \in S} \operatorname{deg}_{H}(v)\right)+k \sum_{\substack{S \subseteq V(H),|S|=k \\
d_{H}(S) \geq k}}\left(\sum_{v \in S} \operatorname{deg}(v)\right) \\
& +a k \sum_{\substack{S \subseteq V(H) \\
|S|=k}} d_{G \vee H}(S) \\
= & k \sum_{\substack{S \subseteq V(H) \\
|S|=k}}\left(\sum_{v \in S} \operatorname{deg}_{H}(v)\right)-\sum_{\substack{S \subseteq V(G),|S|=k \\
\bar{d}_{H}(S)=k-1}}\left(\sum_{v \in S} \operatorname{deg}_{H}(v)\right)+a k\left[k\left(\begin{array}{l}
b \\
k
\end{array}\right)-x\right]
\end{aligned}
$$




$$
=2 k e(H)\left(\begin{array}{l}
b-1 \\
k-1
\end{array}\right)-\sum_{i=1}^{x}\left(\sum_{v \in S_{i}} \operatorname{deg}_{H}(v)\right)+a k^{2}\left(\begin{array}{l}
b \\
k
\end{array}\right)-a k x
$$

Suppose $S \cap V(G) \neq \varnothing$ and $S \cap V(H) \neq \varnothing$. In this case, $d_{G \vee H}(S)=k-1$. Then this case contributes to $S D D_{k}(G \vee H)$ by

$$
\begin{aligned}
& \sum_{\substack{S \subseteq V(G),|S|=k \\
S \cap V(G) \neq \varnothing, S \cap V(H) \neq \varnothing}}\left(\sum_{v \in S} \operatorname{deg}_{G \vee H}(v)\right) d_{G \vee H}(S) \\
= & (k-1) \sum_{\substack{S \subseteq V(G),|S|=k \\
S \cap V(G) \neq \varnothing, S \cap V(H) \neq \varnothing}}\left(\sum_{v \in S} \operatorname{deg}_{G \vee H}(v)\right) \\
= & (k-1) \cdot 2 e(G \vee H)\left(\begin{array}{c}
a+b-1 \\
k-1
\end{array}\right)-(k-1) \cdot 2 e(G)\left(\begin{array}{l}
a-1 \\
k-1
\end{array}\right) \\
& -(k-1) a b\left(\begin{array}{l}
a-1 \\
k-1
\end{array}\right)-(k-1) \cdot 2 e(H)\left(\begin{array}{l}
b-1 \\
k-1
\end{array}\right)-(k-1) a b\left(\begin{array}{l}
b-1 \\
k-1
\end{array}\right) \\
= & (k-1)(2 e(G)+2 e(H)+2 a b)\left(\begin{array}{c}
a+b-1 \\
k-1
\end{array}\right)-(k-1) \cdot 2 e(G)\left(\begin{array}{l}
a-1 \\
k-1
\end{array}\right) \\
& -(k-1) a b\left(\begin{array}{l}
a-1 \\
k-1
\end{array}\right)-(k-1) \cdot 2 e(H)\left(\begin{array}{l}
b-1 \\
k-1
\end{array}\right)-(k-1) a b\left(\begin{array}{l}
b-1 \\
k-1
\end{array}\right) .
\end{aligned}
$$

From the above arguments, we conclude that

$$
\begin{aligned}
& S D D_{k}(G \vee H)= a k^{2}\left(\begin{array}{l}
b \\
k
\end{array}\right)-a k x+2 e(H)\left(\begin{array}{l}
b-1 \\
k-1
\end{array}\right)-\sum_{i=1}^{x}\left(\sum_{v \in S_{i}} \operatorname{deg}_{H}(v)\right) \\
&+2(k-1)[e(G)+e(H)+a b]\left(\begin{array}{c}
a+b-1 \\
k-1
\end{array}\right) \\
&-(k-1) a b\left(\begin{array}{l}
a-1 \\
k-1
\end{array}\right)-(k-1) a b\left(\begin{array}{l}
b-1 \\
k-1
\end{array}\right)-(k-1) \cdot 2 e(G)\left(\begin{array}{l}
a-1 \\
k-1
\end{array}\right),
\end{aligned}
$$

where $S_{1}, S_{2}, \ldots, S_{x}$ are all the $k$-subsets of $V(H)$ such that $H\left[S_{i}\right](1 \leq i \leq x)$ is connected. 
Case 3. $b<k \leq a+b$. For any $S \subseteq V(G)$ and $|S|=k$, we have $S \cap V(G) \neq \varnothing$ and $S \cap V(H) \neq \varnothing$. In this case, $d_{G \vee H}(S)=k-1$. Then

$$
\begin{aligned}
& S D D_{k}(G \vee H) \\
= & \sum_{\substack{S \subseteq \vee(G) \\
|S|=k}}\left(\sum_{v \in S} \operatorname{deg}_{G \vee H}(v)\right) d_{G \vee H}(S) \\
= & (k-1) \sum_{\substack{S \subseteq V(G) \\
|S|=k}}\left(\sum_{v \in S} \operatorname{deg}_{G \vee H}(v)\right) \\
= & (k-1) \cdot 2 e(G \vee H)\left(\begin{array}{c}
a+b-1 \\
k-1
\end{array}\right)-(k-1) \cdot 2 e(G)\left(\begin{array}{l}
a-1 \\
k-1
\end{array}\right) \\
& -(k-1) a b\left(\begin{array}{l}
a-1 \\
k-1
\end{array}\right)-(k-1) \cdot 2 e(H)\left(\begin{array}{l}
b-1 \\
k-1
\end{array}\right)-(k-1) a b\left(\begin{array}{l}
b-1 \\
k-1
\end{array}\right) \\
= & (k-1)[2 e(G)+2 e(H)+2 a b]\left(\begin{array}{c}
a+b-1 \\
k-1
\end{array}\right)-(k-1) \cdot 2 e(G)\left(\begin{array}{l}
a-1 \\
k-1
\end{array}\right) \\
& -(k-1) a b\left(\begin{array}{c}
a-1 \\
k-1
\end{array}\right)-(k-1) \cdot 2 e(H)\left(\begin{array}{l}
b-1 \\
k-1
\end{array}\right)-(k-1) a b\left(\begin{array}{l}
b-1 \\
k-1
\end{array}\right) \\
=2(k- & 1) e(G)\left[\left(\begin{array}{c}
a+b-1 \\
k-1
\end{array}\right)-\left(\begin{array}{l}
a-1 \\
k-1
\end{array}\right)\right]-(k-1) a b\left[\left(\begin{array}{l}
a-1 \\
k-1
\end{array}\right)+\left(\begin{array}{l}
b-1 \\
k-1
\end{array}\right)\right] \\
& +2(k-1) e(H)\left[\left(\begin{array}{l}
a+b-1 \\
k-1
\end{array}\right)-\left(\begin{array}{l}
b-1 \\
k-1
\end{array}\right)\right] .
\end{aligned}
$$

This completes the proof of the theorem.

\subsection{Cartesian product}

In [15], Gologranc obtained the following lower bound for Steienr distance.

Lemma 2.1. [15] Let $k \geq 2$ be an integer, and let $G, H$ be two connected graphs. Let $S=\left\{\left(g_{1}, h_{1}\right),\left(g_{2}, h_{2}\right), \ldots,\left(g_{k}, h_{k}\right)\right\}$ be a set of distinct vertices of 
$G \square H$. Let $S_{G}=\left\{g_{1}, g_{2}, \ldots, g_{k}\right\}$ and $S_{H}=\left\{h_{1}, h_{2}, \ldots, h_{k}\right\}$. Then

$$
d_{G \square H}(S) \geq d_{G}\left(S_{G}\right)+d_{H}\left(S_{H}\right) .
$$

Mao et al. derived the following results for Steiner distance.

Lemma 2.2. Let $G, H$ be two connected graphs of order $n, m$, respectively. Let $k$ be an integer with $3 \leq k \leq m n$. Let $S=\left\{\left(g_{1}, h_{1}\right),\left(g_{2}, h_{2}\right), \ldots,\left(g_{k}, h_{k}\right)\right\}$ be a set of distinct vertices of $G \square H$. Let $S_{G}=\left\{g_{1}, g_{2}, \ldots, g_{k}\right\}$ and $S_{H}=$ $\left\{h_{1}, h_{2}, \ldots, h_{k}\right\}$. Then

$$
\begin{aligned}
d_{G}\left(S_{G}\right)+d_{H}\left(S_{H}\right) & \leq d_{G \square H}(S) \\
& \leq \min \left\{d_{G}\left(S_{G}\right)+(k-2) d_{H}\left(S_{H}\right), d_{H}\left(S_{H}\right)+(k-2) d_{G}\left(S_{G}\right)\right\} .
\end{aligned}
$$

In this section, we give the upper and lower bounds of $S D D_{k}(G \square H)$.

Theorem 2.2. Let $G, H$ be two connected graphs of order $a, b(a \leq b)$, respectively. Let $k$ be an integer with $3 \leq k \leq a+b$. Then

$$
X \leq S D D_{k}(G \square H) \leq\left\lceil\frac{k-1}{2}\right\rceil X,
$$

where

$$
\begin{gathered}
X=\sum_{i=1}^{k} b\left(\begin{array}{c}
b-1 \\
r_{1}-1
\end{array}\right)\left(\begin{array}{c}
b \\
r_{2}
\end{array}\right) \ldots\left(\begin{array}{c}
b \\
r_{i}
\end{array}\right) S D D_{i}(G)+\sum_{j=1}^{k} a\left(\begin{array}{c}
a-1 \\
s_{1}-1
\end{array}\right)\left(\begin{array}{c}
a \\
s_{2}
\end{array}\right) \ldots\left(\begin{array}{c}
a \\
s_{j}
\end{array}\right) S D D_{j}(H) \\
+2 e(H) \sum_{i=1}^{k} b\left(\begin{array}{c}
b-1 \\
r_{1}-1
\end{array}\right)\left(\begin{array}{c}
b \\
r_{2}
\end{array}\right) \ldots\left(\begin{array}{c}
b \\
r_{i}
\end{array}\right) i S W_{i}(G) \\
+2 e(G) \sum_{j=1}^{k} a\left(\begin{array}{c}
a-1 \\
s_{1}-1
\end{array}\right)\left(\begin{array}{c}
a \\
s_{2}
\end{array}\right) \ldots\left(\begin{array}{c}
a \\
s_{j}
\end{array}\right) j S W_{j}(H), \\
\sum_{p=1}^{i} r_{p}=k, \sum_{q=1}^{j} s_{q}=k, r_{p} \geq 1(1 \leq p \leq i), s_{q} \geq 1(1 \leq q \leq j) .
\end{gathered}
$$

Proof. From the definition of Steiner degree distance, we have

$$
S D D_{k}(G \square H)=\sum_{\substack{S \subseteq V(G \square H) \\|S|=k}}\left(\sum_{(u, v) \in S} \operatorname{deg}_{G \square H}(u, v)\right) d_{G \square H}(S) .
$$


Let $S=\left\{\left(u_{1}, v_{1}\right),\left(u_{2}, v_{2}\right), \ldots,\left(u_{k}, v_{k}\right)\right\}$. Then

$$
\sum_{(u, v) \in S} \operatorname{deg}_{G \square H}(u, v)=\sum_{i=1}^{k} \operatorname{deg}_{G}\left(u_{i}\right)+\sum_{i=1}^{k} \operatorname{deg}_{H}\left(v_{i}\right) .
$$

From Lemma 2.1, we have $d_{G \square H}(S) \geq d_{G}\left(S_{G}\right)+d_{H}\left(S_{H}\right)$, and hence

$$
\begin{aligned}
& S D D_{k}(G \square H) \\
& \geq \sum_{\substack{S \subseteq V(G \square H) \\
|S|=k}}\left(\sum_{i=1}^{k} \operatorname{deg}_{G}\left(u_{i}\right)+\sum_{i=1}^{k} \operatorname{deg}_{H}\left(v_{i}\right)\right)\left[d_{G}\left(S_{G}\right)+d_{H}\left(S_{H}\right)\right] \\
&= \sum_{\substack{S \subseteq V(G \square H) \\
|S|=k}}\left(\sum_{i=1}^{k} \operatorname{deg}_{G}\left(u_{i}\right)\right) d_{G}\left(S_{G}\right)+\sum_{\substack{S \subseteq V(G \square H \\
|S|=k}}\left(\sum_{i=1}^{k} \operatorname{deg}_{H}\left(v_{i}\right)\right) d_{H}\left(S_{H}\right) \\
&+\sum_{\substack{S \subseteq V(G \square H) \\
|S|=k}}\left(\sum_{i=1}^{k} \operatorname{deg}_{H}\left(v_{i}\right)\right) d_{G}\left(S_{G}\right)+\sum_{\substack{S \subseteq V(G \square H) \\
|S|=k}}\left(\sum_{i=1}^{k} \operatorname{deg}_{G}\left(u_{i}\right)\right) d_{H}\left(S_{H}\right) \\
&=\sum_{i=1}^{k} b\left(\begin{array}{c}
b-1 \\
r_{1}-1
\end{array}\right)\left(\begin{array}{c}
b \\
r_{2}
\end{array}\right) \ldots\left(\begin{array}{c}
b \\
r_{i}
\end{array}\right) S D D_{i}(G)+\sum_{j=1}^{k} a\left(\begin{array}{c}
a-1 \\
s_{1}-1
\end{array}\right)\left(\begin{array}{c}
a \\
s_{2}
\end{array}\right) \ldots\left(\begin{array}{c}
a \\
s_{j}
\end{array}\right) S D D_{j}(H) \\
&+2 e(H) \sum_{i=1}^{k} b\left(\begin{array}{c}
b-1 \\
r_{1}-1
\end{array}\right)\left(\begin{array}{c}
b \\
r_{2}
\end{array}\right) \ldots\left(\begin{array}{c}
b \\
r_{i}
\end{array}\right) i S W_{i}(G) \\
&+2 e(G) \sum_{j=1}^{k} a\left(\begin{array}{c}
a-1 \\
s_{1}-1
\end{array}\right)\left(\begin{array}{c}
a \\
s_{2}
\end{array}\right) \ldots\left(\begin{array}{c}
a \\
s_{j}
\end{array}\right) j S W_{j}(H)
\end{aligned}
$$

From Lemma 2.2, we have $d_{G \square H}(S) \leq \min \left\{d_{G}\left(S_{G}\right)+d_{H}\left(S_{H}\right)(k-2), d_{H}\left(S_{H}\right)+\right.$ 


$$
\begin{aligned}
\left.d_{G}\left(S_{G}\right)(k-2)\right\} \leq\left\lceil\frac{k-1}{2}\right\rceil\left[d_{G}\left(S_{G}\right)+d_{H}\left(S_{H}\right)\right], \text { and hence } \\
\quad S D D_{k}(G \square H) \\
\leq \quad\left[\frac{k-1}{2}\right\rceil \sum_{\substack{S \subseteq V(G \square H) \\
|S|=k}}\left(\sum_{i=1}^{k} d e g_{G}\left(u_{i}\right)+\sum_{i=1}^{k} d e g_{H}\left(v_{i}\right)\right)\left[d_{G}\left(S_{G}\right)+d_{H}\left(S_{H}\right)\right] \\
=\quad\left[\frac{k-1}{2}\right\rceil \sum_{i=1}^{k} b\left(\begin{array}{c}
b-1 \\
r_{1}-1
\end{array}\right)\left(\begin{array}{c}
b \\
r_{2}
\end{array}\right) \ldots\left(\begin{array}{c}
b \\
r_{i}
\end{array}\right) S D D_{i}(G)+\frac{k}{2} \sum_{j=1}^{k} a\left(\begin{array}{c}
a-1 \\
s_{1}-1
\end{array}\right)\left(\begin{array}{c}
a \\
s_{2}
\end{array}\right) \\
\quad \ldots\left(\begin{array}{c}
a \\
s_{j}
\end{array}\right) S D D_{j}(H)+k e\left(\begin{array}{c}
H \\
r_{1}
\end{array}\right) \sum_{i=1}^{k} b\left(\begin{array}{c}
b-1 \\
r_{1}-1
\end{array}\right)\left(\begin{array}{c}
b \\
r_{2}
\end{array}\right) \ldots\left(\begin{array}{c}
b \\
r_{i}
\end{array}\right) i S W_{i}(G) \\
+k e(G) \sum_{j=1}^{k} a\left(\begin{array}{c}
a-1 \\
s_{1}-1
\end{array}\right)\left(\begin{array}{c}
a \\
s_{2}
\end{array}\right) \ldots\left(\begin{array}{c}
a \\
s_{j}
\end{array}\right) j S W_{j}(H) .
\end{aligned}
$$

This completes the proof of the theorem.

Remark 2.1. For $k=3$, the lower and upper bounds on $S D D_{k}(G \square H)$ are same $(X)$ in Theorem 2.2.

\section{Conclusion}

The Steiner distance in a graph, introduced by Chartrand et al. is a natural generalization of the concept of classical graph distance. The degree distance seems to have been considered first by Dobrynin and Kochetova, and practically at the same time by Gutman, who used a different name for it. The $k$-center Steiner degree distance introduced very recently. In this paper, we obtain the $k$-center Steiner degree distance of complete and Cartesian product graphs. Investigating the other graph products (corona product, composition, disjunction etc.) are our future work.

Acknowledgment. The authors are much grateful to the anonymous referee 
for his valuable comments on our paper, which have considerably improved the presentation of this paper. The first author was supported by the National Science Foundation of China (Nos. 11601254, 11551001, 11161037, 11461054) and the Science Found of Qinghai Province (Nos. 2016-ZJ-948Q, and 2014ZJ-907). The third author was supported by the Sungkyun research fund, Sungkyunkwan University, 2017, and National Research Foundation of the Korean government with grant No. 2017R1D1A1B03028642.

\section{References}

[1] P. Ali, P. Dankelmann, S. Mukwembi, Upper bounds on the Steiner diameter of a graph, Discr. Appl. Math. 160 (2012) 1845-1850.

[2] P. Ali, S. Mukwembi, S. Munyira, Degree distance and vertex-connectivity, Discr. Appl. Math. 161 (2013) 2802-2811.

[3] P. Ali, S. Mukwembi, S. Munyira, Degree distance and edge-connectivity, Australas. J. Comb. 60 (2014) 50-68.

[4] M. An, L. Xiong, K.C. Das, Two upper bounds for the degree distances of four sums of graphs, Filomat 28 (2014) 579-590.

[5] J. A. Bondy, U. S. R. Murty, Graph Theory, Springer, New York, 2008.

[6] O. Bucicovschi, S. M. Cioabă, The minimum degree distance of graphs of given order and size, Discr. Appl. Math. 156 (2008) 3518-3521.

[7] F. Buckley, F. Harary, Distance in Graphs, Addison-Wesley, Redwood, 1990.

[8] J. Cáceresa, A. Márquezb, M. L. Puertasa, Steiner distance and convexity in graphs, Eur. J. Comb. 29 (2008) 726-736.

[9] G. Chartrand, O. R. Oellermann, S. Tian, H. B. Zou, Steiner distance in graphs, Časopis Pest. Mat. 114 (1989) 399-410. 
[10] P. Dankelmann, O. R. Oellermann, H. C. Swart, The average Steiner distance of a graph, J. Graph Theory 22 (1996) 15-22.

[11] A. Dobrynin, R. Entringer, I. Gutman, Wiener index of trees: theory and application, Acta Appl. Math. 66 (2001) 211-249.

[12] A. A. Dobrynin, I. Gutman, S. Klavžar, P. Žigert, Wiener index of hexagonal systems, Acta Appl. Math. 72 (2002) 247-294.

[13] A. Dobrynin, A. Kochetova, Degree distance of a graph: A degree analogue of the Wiener index, J. Chem. Inf. Comput. Sci. 34 (1994) 10821086.

[14] W. Goddard, O. R. Oellermann, Distance in graphs, in: M. Dehmer (Ed.), Structural Analysis of Complex Networks, Birkhäuser, Dordrecht, 2011, pp. 49-72.

[15] T. Gologranc, Steiner Convex Sets and Cartesian Product, Bull. Malays. Math. Sci. Soc. DOI 10.1007/s40840-016-0312-8.

[16] I. Gutman, On Steiner degree distance of trees, Appl. Math. Comput. 283 (2016) 163-167.

[17] I. Gutman, On two degree-and-distance-based graph invariants, Bull. Acad. Serbe Sci. Arts (Cl. Sci. Math. Natur.), in press.

[18] I. Gutman, B. Furtula, K. C. Das, On some degree-and-distance-based graph invariants of trees, Appl. Math. Comput. 289 (2016) 1-6.

[19] I. Gutman, Y. N. Yeh, S. L. Lee, Y. L. Luo, Some recent results in the theory of the Wiener number, Indian J. Chem. 32A (1993) 651-661.

[20] M. Knor, R. Škrekovski, A. Tepeh, Mathematical aspects of Wiener index, Ars Math. Contemp. 11 (2016) 327-352.

[21] X. Li, Y. Mao, I. Gutman, The Steiner Wiener index of a graph, Discuss. Math. Graph Theory 36 (2016) 455-465. 
[22] X. Li, Y. Mao, I. Gutman, Inverse problem on the Steiner Wiener index, Discuss. Math. Graph Theory 38 (2018) 83-95.

[23] Y. Mao, The Steiner diameter of a graph, Bull. Iran. Math. Soc. 43 (2) (2017) 439-454.

[24] Y. Mao, E. Cheng, Z. Wang, Steiner distance in product networks, arXiv:1703.01410 [math.CO] 2017.

[25] Y. Mao, K. C. Das, Steiner Gutman index, MATCH Commun. Math. Comput. Chem. 79 (3) (2018) 779-794.

[26] Y. Mao, Z. Wang, I. Gutman, Steiner Wiener index of graph products, Trans. Combin. 5 (3) (2016) 39-50.

[27] Y. Mao, Z. Wang, I. Gutman, A. Klobučar, Steiner degree distance, MATCH Commun Math. Comput. Chem. 78 (1) (2017) 221-230.

[28] Y. Mao, Z. Wang, I. Gutman, H. Li, Nordhaus-Gaddum-type results for the Steiner Wiener index of graphs, Discrete Appl. Math. 219 (2017) 167175 .

[29] Y. Mao, Z. Wang, Y. Xiao, C. Ye, Steiner Wiener index and connectivity of graphs, Utilitas Math. 102 (2017) 51-57.

[30] S. Mukwembi, S. Munyira, Degree distance and minimum degree, Bull. Austral. Math. Soc. 87 (2013) 255-271.

[31] O. R. Oellermann, S. Tian, Steiner centers in graphs, J. Graph Theory 14 (1990) 585-597.

[32] K. Pattabiraman, P. Kandan, Generalization of the degree distance of the tensor product of graphs, Australas. J. Comb. 62 (2015) 211-227.

[33] D. H. Rouvray, Harry in the limelight: The life and times of Harry Wiener, in: D. H. Rouvray, R. B. King (Eds.), Topology in Chemistry - Discrete Mathematics of Molecules, Horwood, Chichester, 2002, pp. 1-15. 
[34] D. H. Rouvray, The rich legacy of half century of the Wiener index, in: D. H. Rouvray, R. B. King (Eds.), Topology in Chemistry - Discrete Mathematics of Molecules, Horwood, Chichester, 2002, pp. 16-37.

[35] V. Sheeba Agnes, Degree distance and Gutman index of corona product of graphs, Trans. Comb. 4 (3) (2015) 11-23.

[36] K. Xu, M. Liu, K. C. Das, I. Gutman, B. Furtula, A survey on graphs extremal with respect to distance-based topological indices, MATCH Commun. Math. Comput. Chem. 71 (2014) 461-508.

Yaping Mao,

Department of Mathematics, Qinghai Normal University, Xining, Qinghai 810008, China.

Center for Mathematics and Interdisciplinary Sciences of Qinghai Province,

Xining, Qinghai 810008, China

e-mail: maoyaping@ymail.com

Zhao Wang,

School of Mathematical Sciences, Beijing Normal University,

Beijing 100875, China

e-mail: wangzhao380@yahoo.com

Kinkar Ch. Das,

Department of Mathematics, Sungkyunkwan University,

Suwon 440-746, Republic of Korea.

Email: kinkardas2003@googlemail.com 